\title{
PREVALENCE OF THYROID AUTOANTIBODIES AS DETERMINANT OF RECURRENT PREGNANCY LOSS- A HOSPITAL BASED STUDY
}

\author{
Lakshmi G1 , Hema S. Nair², S. P. Sarma ${ }^{3}$ \\ ${ }^{1}$ Associate Professor, Department of Physiology, Government Medical College, Trivandrum. \\ ${ }^{2}$ Additional Professor, Department of Gynaecology, Government Medical College, Trivandrum. \\ ${ }^{3}$ Senior Scientific Officer, Sri Chitra Institute of Medical Sciences, Trivandrum.
}

ABSTRACT

\section{BACKGROUND}

The aim of this study is to find out the association between thyroid autoantibodies and recurrent pregnancy loss. Settings and design, prospective longitudinal study.

\section{MATERIALS AND METHODS}

A total of 73 pregnant females aged 19 - 40 yrs. up to 12 weeks with h/o recurrent pregnancy loss, attending the Obstetrics Clinic at SAT Hospital selected as cases; 209 pregnant females without any h/o pregnancy loss selected as carriers. Their blood collected for antithyroid antibody estimation. Antithyroid peroxidase electroluminescence immunoassay "ECLIA" done using cobar immunoassay analyser at Regional Cancer Centre.

Statistical Analysis - SPSS version of Windows version 10 used. Association among variables were assessed using chi square test, student ' $\mathrm{t}$ ' test.

\section{RESULTS}

$60.3 \%$ cases have raised thyroid peroxidase antibodies as compared to controls (44\%). The study also showed a significant association between antibody positivity and maternal age and the volume of the thyroid gland. There is no significant association between antibody positivity and the number of miscarriages.

\section{CONCLUSION}

Thyroid autoantibodies serve as a useful marker for cases with recurrent pregnancy loss.

\section{KEYWORDS}

Pregnant Women, Thyroid Autoantibodies, Recurrent Pregnancy Loss, Thyroid Volume, Maternal Age.

HOW TO CITE THIS ARTICLE: Lakshmi G, Nair HS, Sarma SP. Prevalence of thyroid autoantibodies as determinant of recurrent pregnancy loss - a hospital-based study. J. Evolution Med. Dent. Sci. 2016;5(97):7105-7110, DOI: 10.14260/jemds/2016/1609

\section{BACKGROUND}

A healthy well-functioning thyroid gland is important for women in their childbearing years. Thyroid disorders are the second most common endocrinologic disorders found in pregnancy. Overt hypothyroidism occur in $0.3 \%-0.5 \%$ of pregnancies, subclinical hypothyroidism in $2-3 \%$,

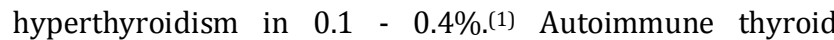
dysfunction remain a common cause of both hypothyroidism and hyperthyroidism in pregnant women.(1) In the developed world, thyroid autoimmunity is the main cause of hypothyroidism, which itself results in poor obstetric outcomes. Thyroid Autoimmunity (TAI) is the most common autoimmune disorder in women of reproductive age with a prevalence varying between 5 and $15 \%$. It is five- to ten-times more common in women than in men and can be present without thyroid dysfunction, thus remaining undiagnosed. The effects of oestrogens in promoting autoimmunity, genetic factors and perhaps maternal microchimerism and

Financial or Other, Competing Interest: None.

Submission 17-10-2016, Peer Review 23-11-2016,

Acceptance 29-11-2016, Published 05-12-2016.

Corresponding Author:

Dr. Lakshmi G,

Associate Professor

Department of Physiology,

Government Medical College, Trivandrum.

E-mail: lakshmi.syam92@gmail.com

DOI: $10.14260 /$ jemds/2016/1609

\section{(c) (i) $(\Theta$}

chromosome $\mathrm{X}$ abnormalities, may potentially explain the high prevalence of TAI in women. Pregnancy is associated with profound changes in thyroid function. The requirements for thyroid hormone increase, a process mediated by the direct thyrotropic effects of human chorionic gonadotropin (hCG), a rise in the binding capacity of serum (Thyroxine-binding globulin) and possibly alterations in the peripheral metabolism of thyroid hormones. Even in women with biochemically normal thyroid function, studies have reported an association between the presence of thyroid autoantibodies, particularly thyroid peroxidase antibodies and adverse pregnancy outcomes including miscarriage, preterm birth and adverse neurodevelopmental sequelae in children. Maternal hyperthyroidism and hypothyroidism affect the outcome of pregnancy producing a higher incidence of miscarriage, infection, preeclampsia, preterm delivery, congestive cardiac failure, thyroid storm and placental abruption. Foetal malformations include prematurity, small size for gestational age, intrauterine foetal death, foetal and neonatal goitre and thyrotoxicosis. $(2,3)$ Even slightly disturbed thyroid dysfunction as subclinical hypothyroidism is associated with increased miscarriages and foetal loss. Since the high prevalence of thyroid autoantibodies in women of reproductive age group, these increases in miscarriage and preterm birth are clinically highly relevant at the individual and population level. Given that minor perturbations in thyroxine concentrations within the normal range can lead to an association between thyroid autoantibodies and adverse 
pregnancy outcomes, trials have been conducted to evaluate the effects of supplementation with levothyroxine on pregnancy outcomes in women with normal thyroid function who tested positive for thyroid autoantibodies.

Thyroid antibodies are autoantibodies targeted against one or more components of thyroid-anti-thyroid peroxidase antibodies, thyrotropin receptor antibodies and antithyroglobulin antibodies. Antithyroid peroxidase antibodies are specific for autoantigen. Thyroid peroxidase is a glycoprotein with molecular weight of 100 - 107KD present on the thyroid cell surface and is important antigenic target in autoimmune thyroid disease. It is responsible for the iodination of tyrosine residues of thyroglobulin and the intramolecular coupling reaction of iodinated tyrosine leading to the formation of triiodothyronine and thyroxine.(4,5) Most antibodies are directed to conformational epitopes of the immunogenic carboxyl terminal region of the TPO protein. Autoimmunity is an important risk factor for miscarriage and preterm birth.(6) Euthyroid pregnant women who are positive for thyroid peroxidase antibodies develop impaired thyroid function, which is associated with an increased risk of miscarriage and premature deliveries.(7)

Miscarriage, the loss of pregnancy before 24 weeks of gestation, affects up to one in five women who conceive, making it the commonest complication of pregnancy. In an "unselected" population of women, the prevalence of thyroid autoantibodies ranges from $6 \%$ to $20 \%$ (40 - 45), being even higher in women with a history of recurrent pregnancy loss at around $17-33 \%$ (32 - 34), and in women with a history of subfertility at around $10-31 \% .(8-10)$

According to American Thyroid Association, $10-20 \%$ of all pregnant women in the first trimester of pregnancy are positive for thyroid autoantibodies, but they are euthyroid.(3)

Recurrent abortion occurs in $0.5 \%$ of all women. Recurrent abortion, typically defined as three or more spontaneous miscarriages, its aetiology is multifactorial and includes uterine anomalies, endocrine disorders, genetic defects and the anticardiolipin antibody. But the cause of pregnancy termination remains unknown in approximately $50 \%$ of the women despite extensive evaluation.(6) High TPOAb levels in pregnant euthyroid women increase the risk of spontaneous miscarriage and preterm birth.(11,12)

Several authors (Stagnaro - Green et al 1990; Glinoer et al 1991; Lejeunne et al 1993; Pratt et al 1993; Kutteh et al 1999;) suspected a link between the presence of thyroid autoantibodies and either isolated or recurrent pregnancy loss.(7) But Espin et al showed that there is no difference in positivity for antithyroid antibodies in non-pregnant women with and without history of recurrent miscarriages. Wilson et al in his study showed that thyroid peroxidase titre and avidity was significantly higher in women with recurrent miscarriages compared to women with recurrent abortion whose pregnancy continued till term with reduction in thyroid autoantibodies titre and avidity. Also Robert et al showed that women with recurrent miscarriage had a higher incidence of thyroid antibodies suggesting an association between thyroid autoimmunity and miscarriage. Despite years of compelling research confirming and explaining upon the significant correlation between high levels of thyroid autoantibodies and an increased rate of miscarriage, many doctors do not test a pregnant women for thyroid autoantibodies.(2) Treatment with levothyroxine in these women seems to decrease the miscarriage rate; it is not yet recommended to treat pregnant euthyroid women with positive antibody test results.(11,12)

Thyroid autoantibodies are common in reproductive age group and thyroid autoimmunity might be associated with miscarriages in women even with normal thyroid function. In fact thyroid autoantibodies has been suggested as one of the markers of at-risk pregnancy.(13,14) Thyroid dysfunction and antibodies during pregnancy seem to predict later thyroid disease.(15) This gap in knowledge regarding whether thyroid autoantibodies is a determinant of recurrent miscarriages in pregnant females in first trimester has prompted me to take up the study.

\section{MATERIALS AND METHODS}

\section{Selection of Patients}

73 pregnant females, aged 19 - 40 yrs. upto 12 weeks attending the Obstetrics Clinic at SAT Hospital with h/o three or more recurrent pregnancy loss were selected as cases. Control group comprise of 209 pregnant females aged 19 - 40 yrs. upto 12 weeks without any h/o pregnancy loss. About $3 \mathrm{~mL}$ of blood taken under aseptic precautions and transported undisturbed to the lab at RCC, Thiruvananthapuram for antithyroid antibodies-antiperoxidase antibodies estimation. Anti-TPOelectroluminescence immunoassay "ECLIA" using cobas immunoassay analyser at RCC, Thiruvananthapuram.

\section{Parameters Studied}

The following parameters were studied. Thyroid peroxidase antibodies titre, its correlation with age, number of miscarriages, thyroid enlargement and thyroid disease.

\section{Statistical Analysis}

For the entry of statistical data, the computer package used was Microsoft Excel. For analysis, SPSS of Windows version 10 was used.

$P$ value of $<0.01$ was considered highly significant.

$P$ value of $<0.05$ was considered significant.

$\mathrm{P}$ value of $>0.05$ was not considered to be significant statistically.

Association among variables were assessed using Pearson Chi square test, student ' $\mathrm{t}$ ' test.

All the parameters are statistically analysed and the tables are seen below.

\section{RESULTS}

Our systematic review and meta-analysis of the study showed that there is a strong association between the presence of thyroid autoantibodies and poor obstetric outcome as miscarriage.

On the basis of definition that thyroid antibody positivity is represented by values $>16 \mathrm{IU} / \mathrm{mL}$. Out of 73 cases 44 (60.3\%) showed higher TPO values, 29 cases (39.7\%) had normal TPO values (Table 1). Only 92 (44\%) among controls had higher TPO values. This difference is statistically significant $(p=0.017)$. Odds ratio relating higher TPO and recurrent pregnancy loss is 1.93 (95\% C.I:1.12 - 3.32).

Mean TPO values were higher for cases compared to controls in the age group less than 25 years and more than 35 years. But mean TPO values lower for cases compared to controls in the middle age groups $25-29$ and $30-34$. Association between age and TPO is not statistically significant 
( $\mathrm{p}=0.962)$ (Figure 1). But percentages with higher TPO is comparable across the age groups.

Among those with abnormal TPO values 53\% had more than three miscarriages $(\mathrm{p}=0.096)$, which correlates a highly significant association between abnormal TPO (> 16IU/L) and recurrent miscarriages (Table 2). Higher the TPO values, higher the mean number of miscarriage $\{($ TPO $17-34), \mathrm{N}=$ 1.34, (35 - 54), $\mathrm{N}=1.43,(>=54, \mathrm{~N}=1.47)\}$ (Figure 2). Thus, increase in miscarriage rates for women positive for thyroid autoantibodies could not be accounted for not only due to the difference in age, but also due to the autoantibodies.

\section{DISCUSSION}

Several mechanisms have been suggested to explain the role of thyroid autoantibodies in recurrent miscarriages.

Firstly, thyroid autoantibodies affect the maternal thyroid gland. The presence of thyroid autoantibodies in women with normal thyroid function could be associated with a subtle deficiency in the availability of thyroid hormones (a fall in circulating free thyroid hormones within the reference range) or a lower capacity of the thyroid gland to adequately rise to the demand for augmented synthesis of thyroid hormones required in pregnancy. $(16,17)$

Secondly, thyroid autoantibodies may cross the placenta and can affect the foetus where $40 \%$ of neonates born to mothers with elevated TPOAb titres at birth. They might be an indicator of an underlying enhanced global autoimmune state. This itself can have a direct adverse effect on placental or foetal development. They can affect the maternal gestational tissues like antiphospholipid antibodies, which cause decidual vascularity and placental insufficiency, which supports the study done by Kennedy R. L. et al.(18)

Thirdly, the thyroid autoantibodies may be the result rather than the cause of recurrent pregnancy loss. This can happen as a consequence of early immune interaction resulting in rejection of the foetus.(19)

Fourth, an interaction exists between thyrotropin like hormones produced by human placenta (Human Chorionic Gonadotropin and Human Chorionic Thyrotropin) and the thyroid autoantibodies resulting in early pregnancy loss.

Fifth antithyroid antibodies reflect generalised activation of the immune system. Stagnaro-Green et al suggested that the autoantibodies are secondary markers of autoimmune risk rather than the specific causative factors. However, the role of thyroid autoantibody positivity as a causative factor of abortion still needs more explanation.(20,21)

Pregnancy is an inflammatory process involving a shift in the regulation of cytokine networks within the local placentaldecidual environment. Dysregulation of local inflammatory process can be associated with miscarriage and premature delivery. The presence of thyroid autoantibodies reflect a dysregulated activity of the immune system at the foetomaternal interface. The proposed mechanisms that predisposes an individual to develop autoimmune thyroid disease include a tissue specific defect in suppressor T-cell activity, a genetically programmed destruction of a thyroid specific antigen and an idiotype/anti-idiotype reaction. Regardless of the cause, the common outcome is the production of 1 or more types of autoantibodies. This itself can have a direct adverse effect on placental or foetal development.(22)
Mean TPO is significantly different between those with thyroid enlargement and without enlargement. This is true for both cases and controls. Mean TPO is significantly different between those with thyroid disease and without thyroid disease (Table 3 - 6).

In the present study thyroid function was different in antibody positive and antibody negative cases, thus a possible influence of mild degree of thyroid insufficiency on incidence of abortion was suggested. It was reported that thyroid antibody positivity often has diminished functional thyroid reserve. This can be explained by the influence of thyroid hormones on angiogenic growth factors and cytokine production as well as trophoblastic proliferation, survival and invasion. Furthermore, the presence of thyroid autoantibodies might be a marker of underlying subtle alteration in thyroid reserve. A reduction in the functional reserve of the thyroid gland associated with reduced adaptation to physiological changes of pregnancy could contribute to minor changes in circulating thyroid hormone concentrations within the reference range. So if there is thyroid enlargement or thyroid disease, TPO antibodies will be high irrespective of whether it is a case or control.(23,24)

Since the thyroid autoantibodies seem to be distinct and independent markers for miscarriages, their identification provides the opportunity to identify pregnant women at risk for an adverse outcome. While many epidemiological studies have investigated the links between thyroid antibodies and pregnancy complications, further randomised clinical trials are needed to investigate the effects of treating pregnant euthyroid women with positive thyroid antibodies on the maternal and early/late neonatal outcomes. $(25,26)$ Therefore, it is suggested to include the determination of thyroid antibodies in the evaluation of women with recurrent miscarriages. The early identification of such pregnancies may eventually provide the opportunity for intervention and prevention of adverse outcomes.

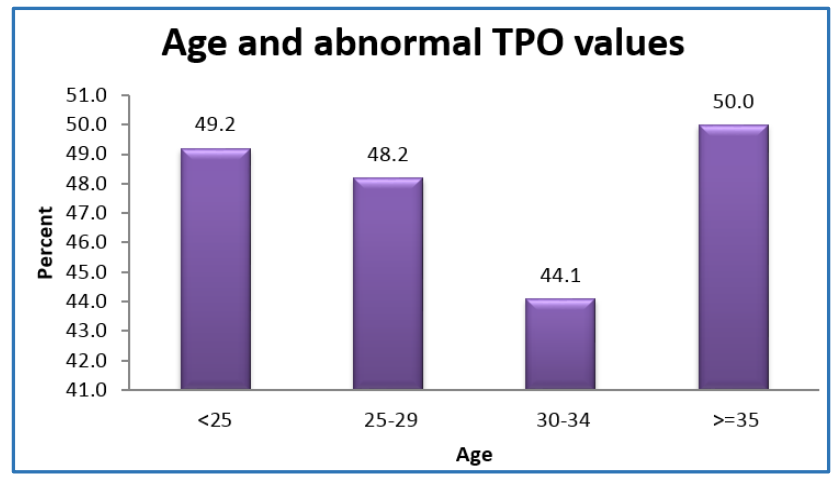

\begin{tabular}{|c|c|c|c|c|c|}
\hline & & & \multicolumn{2}{|c|}{ Case Control } & \\
\hline & & & Control & Case & Total \\
\hline \multirow{4}{*}{ TP0 } & $\begin{array}{c}\text { Normal } \\
1-16\end{array}$ & Count & 117 & 29 & 146 \\
\cline { 2 - 6 } & $\begin{array}{c}\text { \% Within Case } \\
\text { Control }\end{array}$ & $56.0 \%$ & $39.7 \%$ & $51.8 \%$ \\
\cline { 2 - 6 } & Abnormal $>16$ & Count & 92 & 44 & 136 \\
\hline & $\begin{array}{c}\text { \% Within Case } \\
\text { Control }\end{array}$ & $44.0 \%$ & $60.3 \%$ & $48.2 \%$ \\
\hline \multirow{5}{*}{ Total } & $\begin{array}{c}\text { Count } \\
\text { Cithin Case } \\
\text { Control }\end{array}$ & $100.0 \%$ & $100.0 \%$ & $100.0 \%$ \\
\hline & \multicolumn{5}{|c|}{ Table 1. Crosstabs } \\
\hline
\end{tabular}

Chi-Square Test 


\begin{tabular}{|c|c|c|c|}
\hline & Value & df & P Value \\
\hline Pearson Chi-Square & $5.725^{\mathrm{a}}$ & 1 & 017 \\
\hline
\end{tabular}

Abnormal TPO is higher among cases (60.3\%) compared to controls $(44.0 \%)$ and is statistically significant $(\mathrm{P}=0.017)$.

\begin{tabular}{|c|c|c|c|}
\hline \multicolumn{4}{|c|}{ Risk Estimate } \\
\hline & & $95 \%$ Confi & Odds Ratio \\
\hline & Odds Ratio & Lower & Upper \\
\hline Odds Ratio for TPO (0 Normal 1 - 16/1 Abnormal > 16) & 1.930 & 1.122 & 3.320 \\
\hline
\end{tabular}

Odds ratio relating abnormal TPO and recurrent pregnancy loss is 1.93 (95\% CI: 1.12 - 3.32).

\begin{tabular}{|c|c|c|c|c|c|}
\hline & & & \multicolumn{2}{|c|}{ TPO } & \multirow[b]{2}{*}{ Total } \\
\hline & & & 0 Normal 1 - 16 & 1 Abnormal > 16 & \\
\hline \multirow[t]{15}{*}{ No. of Miscarriages } & $1 \mathrm{Nil}$ & Count & 117 & 92 & 209 \\
\hline & & \% Within No. of Miscarriages & $56.0 \%$ & $44.0 \%$ & $100.0 \%$ \\
\hline & & \% Within TPO & $80.1 \%$ & $67.6 \%$ & $74.1 \%$ \\
\hline & 2.3 & Count & 20 & 34 & 54 \\
\hline & & $\%$ Within No. of Miscarriages & $37.0 \%$ & $63.0 \%$ & $100.0 \%$ \\
\hline & & \% Within TPO & $13.7 \%$ & $25.0 \%$ & $19.1 \%$ \\
\hline & 3.4 & Count & 6 & 7 & 13 \\
\hline & & \% Within No. of Miscarriages & $46.2 \%$ & $53.8 \%$ & $100.0 \%$ \\
\hline & & \% Within TPO & $4.1 \%$ & $5.1 \%$ & $4.6 \%$ \\
\hline & $4>=5$ & Count & 3 & 3 & 6 \\
\hline & & \% Within No. of Miscarriages & $50.0 \%$ & $50.0 \%$ & $100.0 \%$ \\
\hline & & \% Within TPO & $2.1 \%$ & $2.2 \%$ & $2.1 \%$ \\
\hline & Total & Count & 146 & 136 & 282 \\
\hline & & $\%$ Within No. of Miscarriages & $51.8 \%$ & $48.2 \%$ & $100.0 \%$ \\
\hline & & \% Within TPO & $100.0 \%$ & $100.0 \%$ & $100.0 \%$ \\
\hline \multicolumn{6}{|c|}{ Table 2. No. of Miscarriages and TPO } \\
\hline
\end{tabular}

Chi-Square Tests

Pearson Chi-Square

a. 2 cells $(25.0 \%)$ have expected count less than 5 . The minimum expected count is 2.89 .

\begin{tabular}{|l|c|c|}
\hline Value & df & P Value \\
\hline $6.350^{\mathrm{a}}$ & 3 & 096 \\
\hline
\end{tabular}

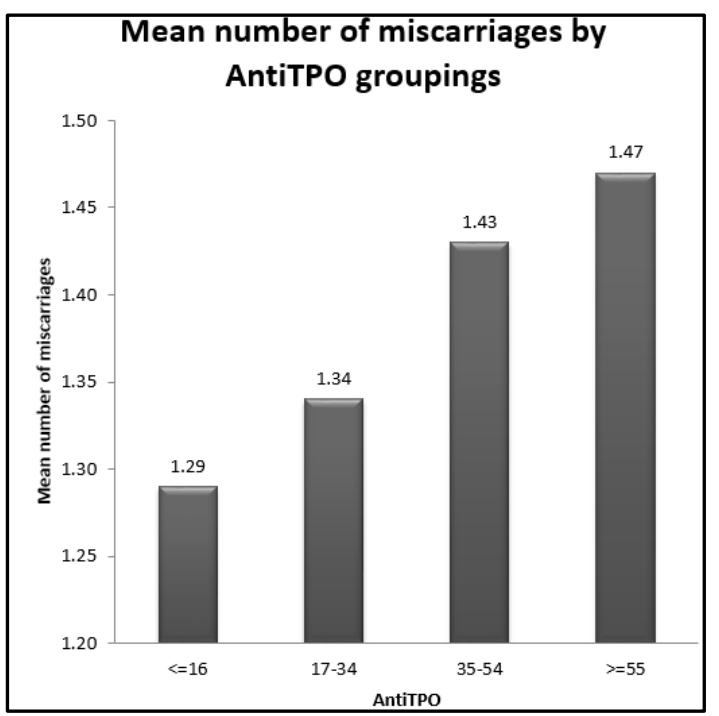

Reports*

AntiTPO

\begin{tabular}{|c|c|c|c|c|c|c|}
\hline $\begin{array}{c}\text { Thyroid_ } \\
\text { Disease }\end{array}$ & Mean & N & Std. Deviation & Median & Minimum & Maximum \\
\hline 0 No & 55.547 & 204 & 123.6555 & 15.300 & 5.0 & 600.0 \\
\hline 1 Yes & 230.580 & 5 & 253.9124 & 109.000 & 13.4 & 516.0 \\
\hline Total & $\mathbf{5 9 . 7 3 5}$ & $\mathbf{2 0 9}$ & $\mathbf{1 2 9 . 9 2 9 9}$ & $\mathbf{1 5 . 3 0 0}$ & $\mathbf{5 . 0}$ & $\mathbf{6 0 0 . 0}$ \\
\hline \multicolumn{7}{|c|}{ Table 3. Anti-TPO Thyroid Disease among Controls Anti-TPO * Thyroid Disease } \\
\hline
\end{tabular}

a. Case Control $=0$ Control

$\mathrm{P}$ value $=.003$
AntiTPO *Thyroid Enlargement

Report*

AntiTPO 


\begin{tabular}{|c|c|c|c|c|c|c|}
\hline Thyroid Enlargement & Mean & N & Std. Deviation & Medium & Minimum & Maximum \\
\hline 0 No & 57.856 & 207 & 126.9332 & 15.300 & 5.0 & 600.0 \\
\hline 1 Yes & 254.200 & 2 & 340.5426 & 254.200 & 13.4 & 495.0 \\
\hline Total & $\mathbf{5 9 . 7 3 5}$ & $\mathbf{2 0 9}$ & $\mathbf{1 2 9 . 9 2 9 9}$ & $\mathbf{1 5 . 3 0 0}$ & $\mathbf{5 . 0}$ & $\mathbf{6 0 0 . 0}$ \\
\hline \multicolumn{7}{|c|}{ Table 4. Anti-TPO and Thyroid Enlargement among Control } \\
\hline
\end{tabular}

a. Case Control $=0$ Control

$\mathrm{P}=.033$

Anti-TPO *Thyroid Disease

Report*

Anti-TPO

\begin{tabular}{|c|c|c|c|c|c|c|}
\hline Thyroid_Disease & Mean & N & Std. Deviation & Median & Minimum & Maximum \\
\hline 0 No & 58.523 & 70 & 101.0874 & 20.495 & 5.0 & 544.0 \\
\hline 1 Yes & 313.767 & 3 & 295.9902 & 332.400 & 8.9 & 600.0 \\
\hline Total & $\mathbf{6 9 . 0 1 2}$ & $\mathbf{7 3}$ & $\mathbf{1 2 1 . 7 7 6 5}$ & $\mathbf{2 0 . 8 9 0}$ & $\mathbf{5 . 0}$ & $\mathbf{6 0 0 . 0}$ \\
\hline \multicolumn{7}{|c|}{ Table 5. Anti-TPO and Thyroid Disease among Cases } \\
\hline
\end{tabular}

a. Case Control $=1$ Case

$\mathrm{P}<.001$

Anti-TPO *Thyroid Enlargement

Report*

Anti-TPO

\begin{tabular}{|c|c|c|c|c|c|c|}
\hline Thyroid Enlargement & Mean & N & Std. Deviation & Medium & Minimum & Maximum \\
\hline 0 No & 61.637 & 72 & 104.9387 & 20.495 & 5.0 & 544.0 \\
\hline 1 Yes & 600.000 & 1 &. & 600.000 & 600.0 & 600.0 \\
\hline Total & 69.012 & 73 & 121.7765 & 20.890 & 5.0 & 600.0 \\
\hline \multicolumn{7}{|c|}{ Table 6. Anti-TPO and Thyroid Enlargement among Cases } \\
\hline
\end{tabular}

a. Case Control $=1$ case

$\mathrm{P}<.001$

\section{CONCLUSIONS}

Thyroid autoantibodies are relatively common in women of reproductive age. Thyroid autoimmunity is associated with adverse pregnancy outcome as miscarriage. Mean TPO values higher for pregnant women in first trimester with h/o recurrent miscarriages compared to controls. The risk of miscarriage is doubled in pregnant women with $\mathrm{h} / \mathrm{o}$ recurrent miscarriages. There is thyroid disease or enlargement of thyroid gland in pregnant women with high TPO values. Since prevalence of thyroid autoimmunity is high among pregnant women of reproductive age group, this problem should be addressed immediately by appropriate detection and early treatment to avoid deleterious effects on mother and offspring.

\section{Limitations of the Study}

The study is of short duration. We were not able to analyse other thyroid autoantibodies, demographic profile of the cases or control due to lack of facilities.

\section{Acknowledgement}

My sincere thanks to all faculty of Gynaecology Department at SAT Hospital, especially my classmate Dr. Hema S. Nair, for full support given for the study in spite of their busy schedule. I take this opportunity to thank Dr. Sankara Sarma, Professor, Sri Chitra Institute of Medical Sciences for giving valuable guidance at different stages of my research work. I sincerely thank the lab technicians in Gynaecology Department for helping me to conduct these tests. My heartfelt thanks to one and all.

\section{REFERENCES}

1. Aziz SFA, Moussa AA, Kandil HO, et al. Antithyroid autoantibodies in unexplained recurrent abortion. Antithyroid 2002;89(1):21-3.

2. Bagis T, Gokcel A, Saygili ES. Autoimmune thyroid disease in pregnancy and the postpartum period: relationship to spontaneous abortion. Thyroid 2001;11(11):1049-53.

3. Bellver J, Soares SR, Alvarez C, et al. The role of thrombophilia and thyroid autoimmunity in unexplained infertility, implantation failure and recurrent spontaneous abortion. Human Reproduction 2008;23(2):278-84.

4. Geva E, Amit A, Lerner-Geva L, et al. Autoimmune disorders: another possible cause for in-vitro fertilization and embryo transfer failure. Human Reprod 1995;10(10):2560-3.

5. Geva E, Amit A, Lerner-Gera L, et al. Autoimmunity and reproduction. Fertil Steril 1997;67(4):599-611.

6. Bussen S, Steck T. Thyroid autoantibodies in euthyroid non-pregnant women with recurrent spontaneous abortions. Human Reproduction 1995;10(11):2938-40. 
7. Chardès $\mathrm{T}$, Chapal $\mathrm{N}$, Bresson $\mathrm{D}$, et al. The human antithyroid peroxidase autoantibody repertoire in graves and hashimotos autoimmune thyroid diseases. Immunogenetics 2002;54(3):141-57.

8. Prummel MF, Wiersinga WM. Thyroid autoimmunity and miscarriage. Eur J Endocrinol 2004;150(6):751-5.

9. Quenby SM, Farquharson RG. Predicting recurring miscarriage: what is important? Obstet Gyecol 1993;82(1):132-8.

10. Roberts J, Jenkins $C$, Wilson $R$, et al. Recurrent miscarriage is associated with increased number of CD5/20 positive lymphocytes and incidence of thyroid antibodies. Eur J Endocrinol 1996;134(1):84-6.

11. Branch DW. Autoimmunity and pregnancy loss. JAMA 1990;246(11):1453-4.

12. Glinoer D, de Nayer P, Bourdoux P, et al. Regulation of maternal thyroid during pregnancy. J Clin Endocrinol Metab 1990;71(2):276-87.

13. Iijima $T$, Tada $H$, Hidaka $Y$, et al. Effects of autoantibodies on the course of pregnancy and fetal growth. Obstetrics and Gynecology 1997;90(3):364-9.

14. Matalon ST, Blank M, Levy Y, et al. The pathogenic role of anti-thyroglobulin antibody on pregnancy: evidence from an active immunization model in mice. Human Reproduction 2003;18(5):1094-9.

15. Muller AF, Verhoeff A, Mantel MJ, et al. Thyroid autoimmunity and abortion: a prospective study in women undergoing in vitro fertilization. Fertil Steril 1999;71(1):30-4.

16. Rushworth FH, Backos M, Rai R, et al. Prospective pregnancy outcome in untreated recurrent miscarriers with thyroid autoantibodies. Human Reprod 2000;15(7):1637-9.

17. Ponnusamy S, Dayan CM. Thyroid autoantibodies. Endocrinology and Metabolism America 2001;30(2):315-37.
18. Shoenfeld Y, Carp HJA, Molina V, et al. Autoantibodies and prediction of reproductive failure. American Journal of Reproductive Immunology 2006;56(5-6):337-44.

19. Singh A, Danta ZN, Stones SC, et al. Presence of thyroid antibodies in early reproductive failure: biochemical versus clinical pregnancies. Fertil Steril 1995;63(2);27781.

20. Stagnaro-Green A. Thyroid antibodies and miscarriage where are we at a generation later? Journal of Thyroid Research 2011;2:841949.

21. Stagnaro-Green A, Glinoer D. Thyroid autoimmunity and the risk of miscarriage. Best Pract Res Clin Endocrinol Metab 2004;18(2):167-81.

22. Stagnaro-Green A, Roman SH, Cobin RH, et al. Detection of at-risk pregnancy by means of highly sensitive assays for thyroid autoantibodies. JAMA 1990;264(11):1422-5.

23. Thangaratinam S, Tan A, Knox E, et al. Association between thyroid autoantibodies and miscarriage and preterm birth: meta-analysis of evidence. British Medical Journal 2011:342-616.

24. Unnikrishnan AG, Buva CN, Detroja. NM, et al. Throid autoimmunity in euthyroid and hypothyroid pregnancies. Land mark papers in thyroid research \&practice. Journal of the Indian thyroid society 2007;4(1):96-7.

25. Boogaard VDE, Vissenberg R, Land JA, et al. Significance of (sub)clinical thyroid dysfunction and thyroid autoimmunity before conception and in early pregnancy: a systematic review. Human Reproduction Update 2011;17(5):605-19.

26. Wilson R, Ling H, MacLean MA, et al. Thyroid antibody titre and avidity in patients with recurrent miscarriage. Fertil Steril 1999;71(3):558-61. 